\title{
Self-Organized Bike Redistribution in Urban City
}

\author{
Yiheng Chi \\ Shenzhen Foreign Languages School, Shenzhen, China \\ Email: chiyiheng2017@163.com
}

How to cite this paper: Chi, Y.H. (2018) Self-Organized Bike Redistribution in Urban City. American Journal of Operations Research, 8, 386-394. https://doi.org/10.4236/ajor.2018.85022

Received: January 30, 2018

Accepted: September 26, 2018

Published: September 29, 2018

Copyright $\odot 2018$ by author and Scientific Research Publishing Inc. This work is licensed under the Creative Commons Attribution International License (CC BY 4.0).

http://creativecommons.org/licenses/by/4.0/

\begin{abstract}
Bicycle is an affordable and environmental friendly alternative to private cars and public transportation. Recently, some big cities in China established the bike-sharing system (BSS) through which people can rent bikes offered by government or commercial companies. However, due to limited parking space, it is often difficult for bikers to park their bicycles in bike stations. This paper envisions approaching this problem by using a self-organized bike redistribution strategy: as time passes by, bike society will form an equilibrium state of bike redistribution.
\end{abstract}

\section{Keywords}

Bike-Sharing System, Self-Organization, Bike Distribution, Multi-Agent System

\section{Introduction}

Traffic congestion has always been a constant headache for populated cities. Several decades ago, people tried to solve this issue by switching to subways. But as the population grows, it is clear that subway alone cannot tackle the problem. What's more, increasing traffic has caused numerous respiratory diseases, one of the top leading causes of death worldwide. Hence, bikes have become a perfect choice for the modern society. With bikes, people can commute and exercise at the same time. Many big cities, therefore, have installed a bike sharing system. However, despite the countless benefits of a bike sharing system, it is a serious problem for bikers to find available parking spaces.

In a social system like bike sharing distribution, every person can be considered as an agent. Each agent has his or her rule of reasoning and actions. In bike sharing system, every person has the same goal of parking their bikes within 
the shortest possible time. When they have to park their bicycles in a congested bike station, they would be unsatisfied with the bike parking environment and might choose to leave next time when they park their bicycles. As every agent makes his or her own actions based on his or her own preference of the environment situation, the bike sharing system can reach an equilibrium state where people eventually find a comfortable place to park their bikes and the society itself will not experience any more wasted effort among bikers by switching bike stations to park.

In this paper, we analyzed the redistribution of shared bikes parking in urban city flooded with bike stations and bike riders. Then we developed a bike sharing model in Netlogo [1], a common multi-agent simulation software in the multi-agent research field. The model applies the environment setting and the social rules adopted by bike riders. After several iterations of the model, we find the equilibrium of bike stations, agent tolerance and agent happiness. Further analysis of the model suggests that more bike stations and fewer bikes will result in higher biker happiness.

\section{Related Works}

There are numerous literatures which talked about shared-bikes riding. For instance, Preisler introduced a Decentralized Coordination Framework (DeCoF), which is designed based on the concept of self-organizing dynamic [1]. The self-organizing redistribution of bike dynamic helps a system to adapt to both external and internal influences controlled by the decentralized coordination processes. Van Lon established a UML activity diagram that describes the rent/return process from a user's perspective by using RinSim, (an open source discrete time MAS simulator solving transportation and logistics problems.) [2]. In another paper, Zhang, Lihong, et al. indicated that bike sharing is a mode of transport that can be sustainable for not only bicycle users but also suppliers [3]. The authors compared five sets of data gained in five big cities in China and finally concluded that agencies like police can ensure the system to work more smoothly and the most effective business models involve government-led investment.

In another paper which talked about bike sharing, the authors adapted greedy, PILOT construction heuristic, Variable Neighborhood Search (VNS) and GRASP strategies and reached the following conclusion: while VNS is the most powerful for the long run, the dynamic approaches are indeed effective; greedy or PILOT construction heuristics are useful for the short run [4]. Caggiani, Leonardo, and M. Ottomanelli divided the redistribution scheme into "user based" and "operator based" [5]. Furthermore, Decision Support System (DSS) for dynamic bike redistribution was presented and the authors found out that DSS will reduce the number of lost users. Vogel, Patrick, T. Greiser and D.C. Mattfeld employed a Geo BI approach for location planning in BSS [6]. With some case studies and bike-sharing modeling, the authors came to the following result: BSS enhances inner-city public transport options and the stations' location will affect 
the bike activity and the demand of the customers.

Chardon defined a rebalanced effective usage (REU) of stations: stations should be divided into high and low value based on how many trips they generate while adjustments are made to address outages and at the same time prepare for the next peak period [7]. Dubernet came up with a multi-agent activity-based model in order to deal with the shortage of bikes in stations caused by uphill riding [8]. Shaheen reviewed the history of bike-sharing and then presented their prediction of shared bikes in the future [9]. Wang, Xize, et al. used models to analyze the effect of nearby business and jobs on trips to and from stations [10]. They figured out that the most significant factors of bike sharing systems are: race, distance to water (distance to $\mathrm{CBD}$ ), trail existence, and operational date [10].

Chemla, Daniel, et al. focused their research on the imbalance problems generated from the self-service bike sharing system [11]. They reached the conclusion that short-term strategies are more efficient, and operators have to obey the "Keep It Simple \& Stupid principles" [11]. Elliot Fishman reviewed the past works on bike sharing and also brought out some problems that have not been solved yet. For example, bike-sharing system has the ability to stimulate private bike riding, an issue that deserves more attention [12]. Schoner attempted to figure out how people navigate from place to place using the bike sharing system [13]. They investigated people's relative preferences to biking over walking. Also, they found out that factors such as distance to access stations, station amenities and neighborhood attributes can affect people's choice of stations [13].

Shahsavaripour discussed a new approach for developing bike sharing network system that can better increase mobility in urban settings [14]. They first explained the planning process and after a series of discussion, they arrived at the conclusion that the density of stations has to be high enough to make the trips convenient. In [15], the main topic is about how the operators of the bike sharing system can rebalance the system with limited resources. The authors utilized past data and came up with a plan for rebalancing bike distribution during rush hours [15]. The impact of social rules on the performance of a team was explored [16]. The author presented a "search and capture" case study and found out that in organization team building, team size should be made neither too small nor too large to ensure team efficiency. However, as team size grows, the effectiveness of the organization increases [16].

In the previous papers, most of the authors discussed about the strategies that operators can use for rebalancing the bikes. However, to the best of author's knowledge, no past work has looked at bike distribution from a self-organized perspective. In this essay, we will employ Net Logo [1] to study the self-organization behavior of bike sharing system.

\section{Self-Organized System and Social Rule}

\section{1) Self-Organized System}

Self-organized system (SOS) is a system which comprises of individual agents 
with perceptions and actions [17]. In SOS, each agent follows a set of universal rules and makes actions accordingly. At a system level, the system itself is able to achieve complex and emergent behavior.

\section{2) Social Rule}

Social rules are the rules that socially interacting agents like bike users follow in a socially interactive society. In our paper, when each person tries to park their bicycles, they follow a set of social rules:

Social Rule 1: Bike riders are satisfied with the current situation when the number of parked bicycles does not exceed the capacity of the bike station in which their own bike is parked.

Social Rule 2: Bike riders are unsatisfied with the current situation when the number of parked bicycles exceeds the capacity of the bike station in which their own bike is parked.

Associated with the social rules, there are two actions that bike riders adopt depending on their satisfaction status with the current situation:

Action 1: When bike riders are satisfied, they continue to park their bikes in the same bike station.

Action 2: When bike riders are not satisfied, they choose to park their bikes in other bike stations.

Finally, when all the agents within the self-organized system become happy, the simulation will stop and an equilibrium state will be reached where every agent in the society is happy with their situation. In other words, every bike rider is able to find a comfortable situation where they do not have to switch between different bike stations to park their bikes.

\section{Environment Setup and Model Development}

The simulation was conducted on Net Logo platform where we can set up different situation parameters and compare different results. In our simulation, tick is a measurement of simulation time, which is similar to real time spent in a society. We use energy as the measurement of an agent's effort in commuting between different bike stations. In our case, energy is considered an effort that each agent makes in the process of finding a suitable place to park. The capacity of a bike station is denoted as tolerance. Figure 1 shows a screenshot of the simulation environment. In this figure, each wheel shape agent denotes a bicycle that an individual agent uses for commuting. At the bottom, the number shows how many bicycles are parked in each individual station. For example, there are 5 bicycles at the first bike station on the right and 8 bicycles parked at the second bike station from the right.

In Figure 1, the wheels represent the agents and the numbers under them are the "stations", which indicate how many agents are staying in the station.

Bike sharing performance is measured based on three variables:

1) Average-Happy-Agent: This variable is calculated by dividing the sum of number of happy-agent (agent satisfied with the current situation) at every time 


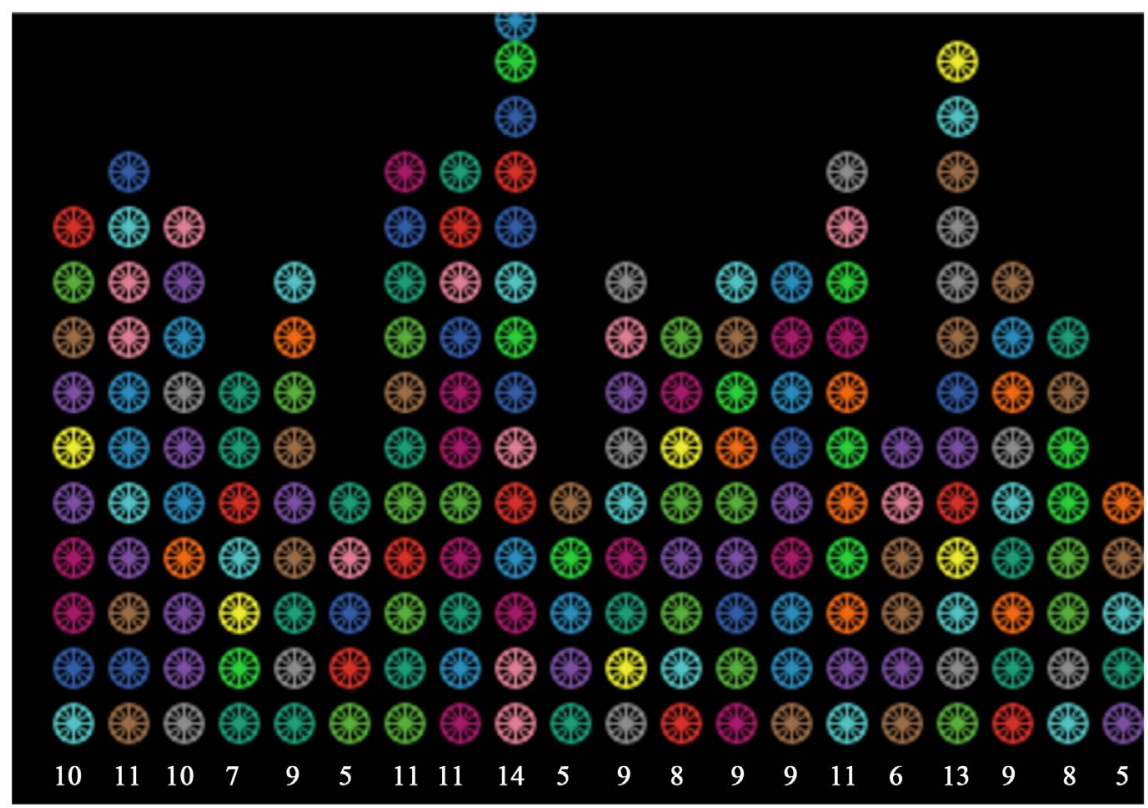

Figure 1. Screenshot of the environment setup of our model based on the NetLogo platform.

step (also called tick) by the total number of time steps (a measurement of simulation time).

2) Ticks: a measure of simulation time in Netlogo.

3) Average energy: This variable is calculated by dividing the total energy of agents by the number of agents. In this model, every time the agents move, they will spend one unit of energy. The more energy an agent makes, the greater effort he spent.

Our simulation has different combinations of settings. Figure 2 shows the number of input variables and output variables we use for the simulations. We change the number of bikes, tolerance and number of stations in the simulation and measure three different performance variables: average-happy-agent, average-energy and ticks.

Table 1 summarizes the combinations of input variables we use for the simulation. In Group 1 simulation, there are 5 bike stations, and each has tolerance 40 and we vary the number of bikes in the society and see how the performance variables change with the input number. Similarly, in Group 2 and Group 3, we change tolerance and number of stations and vary the number of bicycles to get different results of performance variables. In each group of experiments, the number of stations times tolerance all equal to 200 , which is the total capacity of the sum of bike stations. We would like to find the best strategy when designing the bike stations.

\section{Result of Case Study}

Each simulation was run 100 times to maintain the statistical significance of the result and to avoid randomness in the model. 


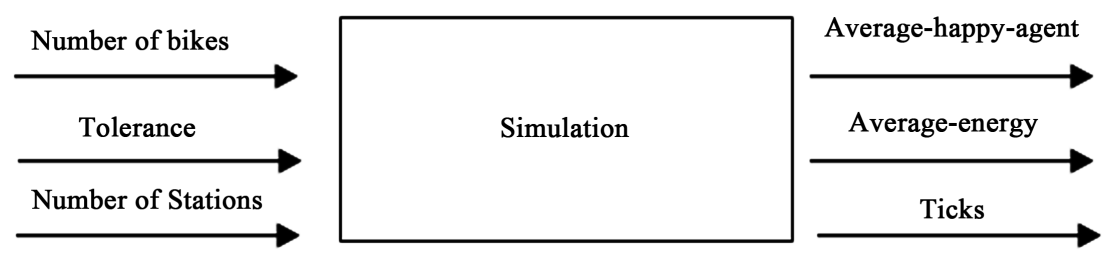

Figure 2. Experiment design with three independent variables and three dependent variables.

Table 1. The summarization of the input variables in our simulation.

\begin{tabular}{cccc}
\hline & \multicolumn{3}{c}{ Input variables and ticks run by the simulation model } \\
\cline { 2 - 4 } & Group 1 & Group 2 & Group 3 \\
\hline Tolerance & 40 & 20 & 10 \\
Number of Stations & 5 & 10 & 20 \\
Ticks & & $150,160,170,180$ & \\
\hline
\end{tabular}

Figure 3 measures the average-happy-agent in a self-organized bike sharing system with different number of bicycles under various numbers of bike stations and tolerances. We can see that when the number of bike station is 5 and tolerance is 40 , increasing the number of bicycles first increase the average happy agent and then as number of bicycles further increase the average happy agent decrease.

However, when the number of bike stations increases but with fewer tolerances, average happy agent has an inverse correlation with the number of bicycles, which means that with more bicycles, there is fewer average number of happy bicycle riders. And this correlation is linear. We should also note that increasing the number of bicycle stations is good for the system to maintain a high number of average happy agents, which means that more people are happy with current bicycle sharing parking status with more bike stations.

Figure 4 shows the average energy of bicycle riders after each simulation run. We found that when bicycle station is 5 with a tolerance of 40 , it is very easy for self-organizing the parking. Little effort was spent by bicycle riders. As the number of bicycles increases, the average energy of bicycle rider can increase dramatically. And this increase is non-linear. Especially when bike station is 20 and tolerance is 10 , with only 170 bicycles present, the average energy of bicycle can increase to a very large number, which means each bicycle rider will spend a significant amount of effort in finding a comfortable place to park their bicycles.

Figure 5 shows the ticks measurement of the bicycle self-organized redistribution under different numbers of bicycles with various numbers of bicycle stations and tolerances. Similar to Figure 4, at all circumstances, increasing the number of bicycles takes more time for system to redistribute. This is more significant when the number of bike stations increases to 20 , with 170 numbers of bicycles, it takes a huge amount of time for the system to reach an equilibrium state. And this correlation is nonlinear and can increase dramatically afterwards if a threshold number of bicycles is reached. 


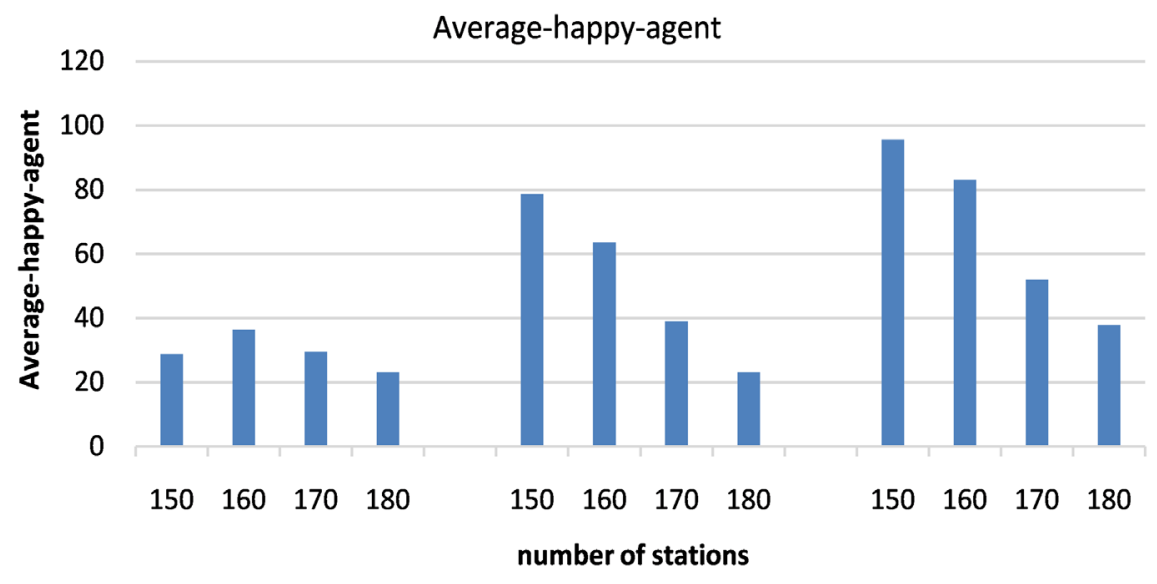

Figure 3. The overall average-happy-agent.

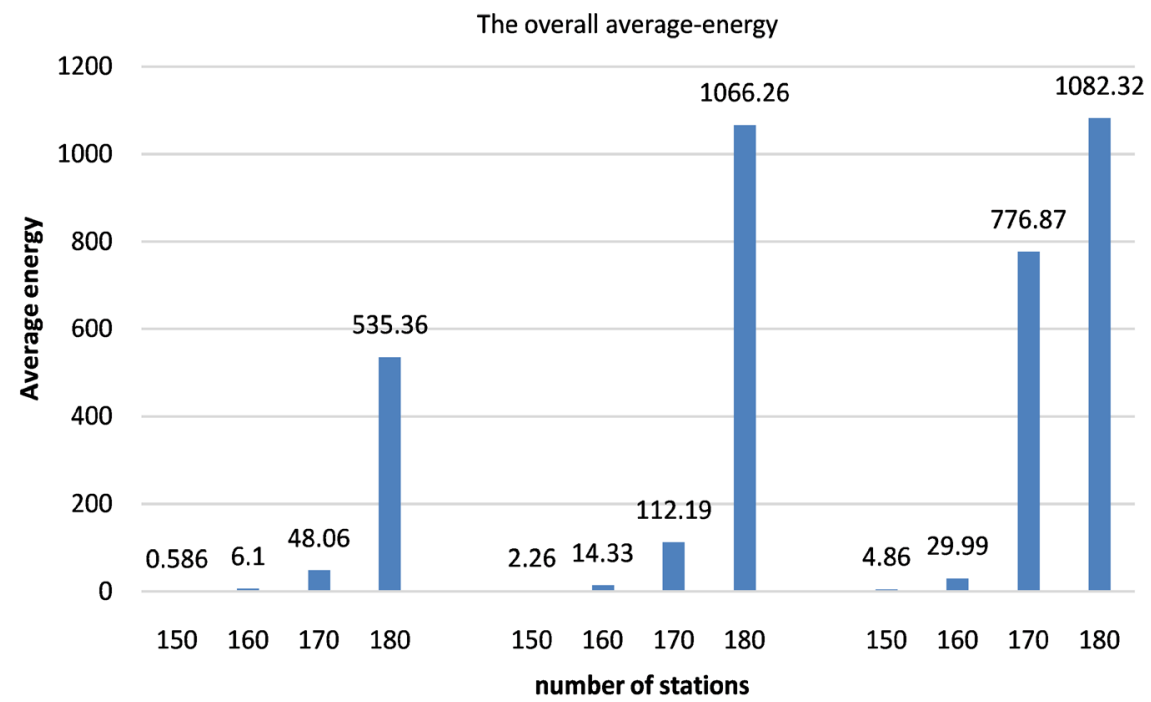

Figure 4. The overall average-energy.

The overall ticks

$$
2500
$$

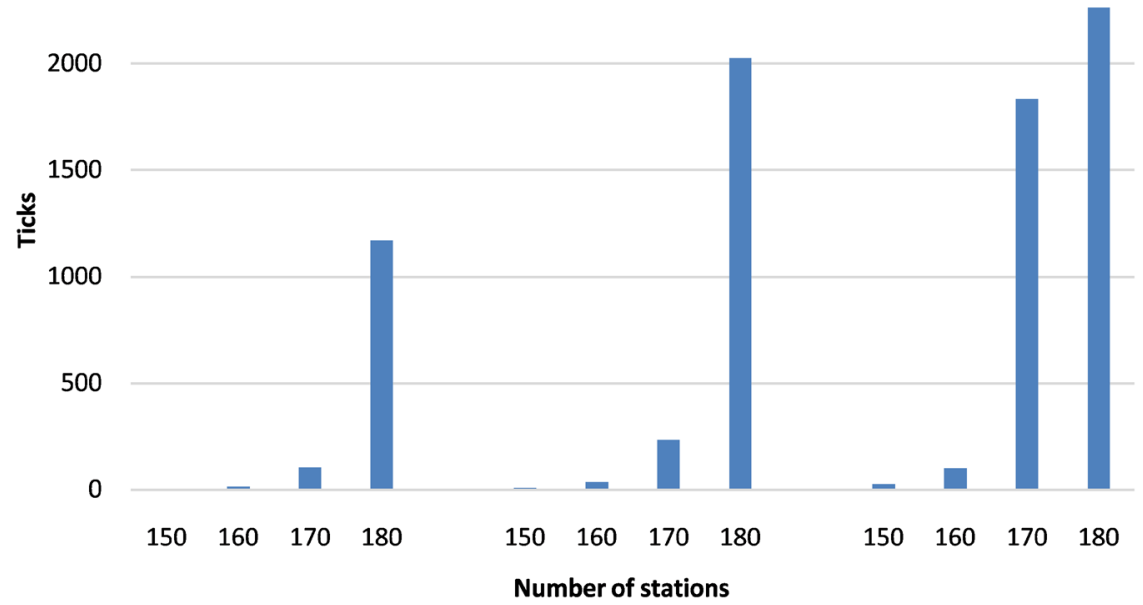

Figure 5. The overall ticks. 


\section{Conclusions and Future Works}

In this paper, a self-organized bike redistribution system was presented. Relevant bike sharing social rule was discussed. The simulation simulated bike sharing society under various inputs and we analyzed results with three different parameters: average-happy-agent, average-energy and ticks. We have reached the following important conclusions for the law and policy makers in designing the bike sharing system (BSS) when the total capability of the sum of tolerance of bike station is equal (limited by resources, budget and etc.):

1) Increasing the number of bicycle stations (but with fewer tolerances) can maintain a high average number of happy bikers who seek suitable parking places.

2) As the number of bicycle stations increases, the average energy of the biker and ticks increase, and this increase is non-linear.

3) Law and policy makers should make sure that the number of bicycles not exceed the threshold because exceeding it would lead to a dramatic increase of both effort made by bicycle riders and time for bike redistribution.

However, there are several limitations of this study. First, we did not consider other elements which may affect the process. For example, the local geography will have a huge effect on the number of bikes in the stations. The stations on the top of a hill always suffer from lack of bikes available and the stations near prosperous regions are always overfilled. How the process works in different situations should be discussed in future study.

Last but not least, the influence of human-redistribution to self-organization of the system is not discussed in this paper. In the future, how these two can work together should be discussed deeply in order to improve the overall bike sharing system.

\section{Conflicts of Interest}

The authors declare no conflicts of interest regarding the publication of this paper.

\section{References}

[1] Thomas, P., Dethlefs, T. and Renz. W. (2016) Self-Organizing Redistribution of Bicycles in a Bike-Sharing System based on Decentralized Control. Federated Conference on Computer Science and Information Systems, Gdansk, 11-14 September 2016, 1471-1480.

[2] Van Lon, R.R.S. and Holvoet, T. (2012) RinSim: A Simulator for Collective Adaptive Systems in Transportation and Logistics. 2012 IEEE Sixth International Conference on Self-Adaptive and Self-Organizing Systems, Lyon, 10-14 September 2012, 231-232. https://doi.org/10.1109/SASO.2012.41

[3] Zhang, L.H., et al. (2015) Sustainable Bike-Sharing Systems: Characteristics and Commonalities across Cases in Urban China. Journal of Cleaner Production, 97, 124-133. https://doi.org/10.1016/j.jclepro.2014.04.006

[4] Kloimüllner, C., et al. (2014) Balancing Bicycle Sharing Systems: An Approach for 
the Dynamic Case. European Conference on Evolutionary Computation in Combinatorial Optimization, Granada, 23-25 April 2014, 73-84.

[5] Caggiani, L. and Ottomanelli, M. (2012) A Modular Soft Computing Based Method for Vehicles Repositioning in Bike-Sharing Systems. Procedia-Social and Behavioral Sciences, 54, 675-684. https://doi.org/10.1016/j.sbspro.2012.09.785

[6] Vogel, P., Greiser, T. and Mattfeld, D.C. (2011) Understanding Bike-Sharing Systems using Data Mining: Exploring Activity Patterns. Procedia-Social and Behavioral Sciences, 20, 514-523. https://doi.org/10.1016/j.sbspro.2011.08.058

[7] De Chardon, C.M., Caruso, G. and Thomas, I. (2016) Bike-Share Rebalancing Strategies, Patterns, and Purpose. Journal of Transport Geography, 55, 22-39. https://doi.org/10.1016/j.jtrangeo.2016.07.003

[8] Dubernet, T. and Axhausen, K.W. (2014) A Multiagent Simulation Framework for Evaluating Bike Redistribution Systems in Bike Sharing Schemes. ArbeitsberichteRaum-und Verkehrsplanung, Zurich, 1010.

[9] Shaheen, S.A., Guzman, S. and Zhang, H. (2010) Bike Sharing in Europe, the Americas, and Asia: Past, Present, and Future. Transportation Research Record Journal of the Transportation Research Board, 2143, 159-167.

https://doi.org/10.3141/2143-20

[10] Wang, X.Z., et al. (2016) Modeling Bike Share Station Activity: Effects of Nearby Businesses and Jobs on Trips to and from Stations. Journal of Urban Planning \& Development, 142, Article ID: 04015001. https://doi.org/10.1061/(ASCE)UP.1943-5444.0000273

[11] Chemla, D., et al. (2013) Self-Service Bike Sharing Systems: Simulation, Repositioning, Pricing.

[12] Fishman, E., Washington, S. and Haworth, N. (2014) Bike Share: A Synthesis of the Literature. Urban Transport of China, 33, 148-165.

[13] Schoner, J. and Levinson, D. (2013) Which Station? Access Trips and Bike Share Route Choice. University of Minnesota Digital Conservancy, Minneapolis.

[14] Shahsavaripour, S. and Shahsavaripour, S. (2015) A New Approach for Developing Bike Sharing, Network System. Journal of Civil Engineering Research, 5, 28-32.

[15] O’Mahony, E. and Shmoys, D.B. (2015) Data Analysis and Optimization for (Citi) Bike Sharing. Twenty-Ninth AAAI Conference on Artificial Intelligence, Austin, 25-30 January 2015, 687-694.

[16] Ji, H. and Jin, Y. (2017) Adoption of Social Rules in Teams of Different Sizes. Engineering Management Reviews, 6, 6-15. https://doi.org/10.14355/emr.2017.0601.002

[17] Simon, H.A. (1982) Models of Bounded Rationality: Empirically Grounded Economic Reason Vol. 3. MIT Press, Cambridge. 